\title{
Quantum effects in an anharmonic crystal
}

\author{
Yu.Kozitsky \\ Institute of Mathematics, Maria Curie-Skłodowska University, \\ 20-031 Lublin, Poland
}

Received August 30, 2002

\begin{abstract}
A model of quantum particles performing $D$-dimensional anharmonic oscillations around their equilibrium positions which form the $d$-dimensional simple cubic lattice $\mathbf{Z}^{d}$ is considered. The model undergoes a structural phase transition when the fluctuations of displacements of particles become macroscopic. This phenomenon is described by susceptibilities depending on Matsubara frequencies $\omega_{n}, n \in \mathbf{Z}$. We prove two theorems concerning the thermodynamic limits of these susceptibilities. The first theorem states that the susceptibilities with nonzero $\omega_{n}$ remain bounded at all temperatures, which means that the macroscopic fluctuations in the model are always non-quantum. The second theorem gives a sufficient condition for the static susceptibility (i.e. corresponding to $\omega_{n}=0$ ) to be bounded at all temperatures. This condition involves the particle mass, the anharmonicity parameters and the interaction intensity. The physical meaning of this result is that, for all $D$ and all values of the temperature, strong quantum effects suppress critical points and the long range order. The proof is performed in the approach where the susceptibilities are represented as functional integrals. A brief description of the main features of this approach is delivered.
\end{abstract}

Key words: displacements, structural phase transition, critical point

PACS: 05.50.-d, 64.60.-i

\section{Introduction}

We consider the following model of anharmonic crystal. To each point $\mathbf{l}$ of the $d$-dimensional simple cubic lattice $\mathbf{Z}^{d}$ there is attached a quantum particle of mass $m$ performing isotropic $D$-dimensional anharmonic oscillations in the crystalline field. The Hamiltonian of our model has the form

$$
\begin{aligned}
H & =\frac{1}{2} \sum_{\mathbf{l}, \mathbf{l}^{\prime} \in \mathbf{Z}^{d}} d_{\mathbf{l}^{\prime}}\left(\mathbf{q}_{\mathbf{l}}, \mathbf{q}_{\mathbf{l}^{\prime}}\right)+\sum_{\mathbf{l} \in \mathbf{Z}^{d}} H_{\mathbf{l}}, \\
H_{\mathbf{l}} & =\frac{1}{2 m}\left(\mathbf{p}_{\mathbf{l}}, \mathbf{p}_{\mathbf{l}}\right)+\frac{k}{2}\left(\mathbf{q}_{\mathbf{l}}, \mathbf{q}_{\mathbf{l}}\right)+V\left(\left(\mathbf{q}_{\mathbf{l}}, \mathbf{q}_{\mathbf{l}}\right)\right), \quad k>0,
\end{aligned}
$$


where $(\ldots, \ldots)$ stands for the scalar product in $\mathbf{R}^{D}$ and $d_{\mathbf{l}}$ is a dynamical matrix of the model. We shall suppose that $d_{1 \mathbf{l}^{\prime}}$ is:

- invariant under translations on $\mathbf{Z}^{d}$;

- ferroelectric, which means $d_{\mathbf{l l}^{\prime}} \leqslant 0$;

- such that $\sum_{\mathbf{l} \in \mathbf{Z}^{d}}\left|d_{\mathbf{l 1}^{\prime}}\right|<\infty$.

These assumptions imply that there exists a function $\phi: \mathbf{R}_{+} \rightarrow \mathbf{R}_{+} \stackrel{\text { def }}{=}[0,+\infty)$, such that

$$
d_{\mathbf{l l}^{\prime}}=-\phi\left(\left|\mathbf{l}-\mathbf{l}^{\prime}\right|\right),
$$

where $\left|\mathbf{l}-\mathbf{l}^{\prime}\right|=\sqrt{\left(l_{1}-l_{1}^{\prime}\right)^{2}+\ldots+\left(l_{d}-l_{d}^{\prime}\right)^{2}}$ is the Euclidean distance on the lattice.

The anharmonicity potential $V$ is supposed to be of the following form

$$
V(\xi)=a \xi+b_{2} \xi^{2}+\ldots+b_{p} \xi^{p}, \quad a \in \mathbf{R}, \quad b_{2}, \ldots, b_{p-1} \geqslant 0, \quad b_{p}>0 .
$$

The simplest case of such a potential is where $p=2[18,27,34]$. If the parameter $a$ is negative and its absolute value is big enough, the equilibria at $\mathbf{q}_{\mathbf{l}}=0, \mathbf{l} \in \mathbf{Z}^{d}$ become unstable (a double-well profile for $D=1$ ) and macroscopic displacements of particles from these unstable positions may occur. Such objects have been studied for many years as quite realistic models of crystalline substance undergoing structural phase transitions [14-16,26,27,34,38]. They, and similar models, are also employed as parts of the models describing strong electron-electron correlations caused by the interaction of electrons with vibrating ions $[18,35,36]$.

Starting from the pioneering paper [31], many efforts were made to show that "the more quantum is the model, the less possible is the phase transition". To confirm this physical common wisdom, different methods and approaches were applied. The first fully rigorous proof of the suppression of the long range order in models of this type with one-dimensional oscillations was done in [40]. This effect was also demonstrated on certain exactly solvable models of anharmonic crystals [29,39].

In [4] it was shown (also for the scalar case) that not only the long range order but any critical anomaly is suppressed if the model is strongly quantum. Later in [22-24] his result was obtained for the vector case with arbitrary $D \in \mathbf{N}$. The present paper gives an extended and complete presentation of the results announced in the latter papers. In section 2 below we formulate two theorems. The physical meaning of the first theorem is that the large fluctuations of displacements of particles, which appear at the critical point, are always non-quantum. This means that the only static susceptibility may diverge. The second theorem states that also the static susceptibility is bounded (i.e. non-quantum large fluctuations are suppressed as well) at all temperatures if the model is strongly quantum. This occurs if either the particles are light and/or the energy levels of the one-particle Hamiltonian of the corresponding scalar model (see below) are sufficiently separated from each other. Both these mechanisms are observed experimentally as isotopic effects (light particles) $[1,38]$ and external hydrostatic pressure effects (big separation) [13,37]. The proof of the second theorem is performed in the approach which employs functional 
integrals, briefly described in section 3. This approach, initiated in [2], now is well elaborated in $[3,8-11,20,25]$. A complete description of this approach in application to the model considered in the present paper and an extended related bibliography are given in the review article [6]. In section 4 we discuss some consequences of our theorems.

\section{The results}

The description of the Gibbs states of lattice models is performed with the help of local Gibbs states, which are constructed by means of local Hamiltonians (see $[8,9,19,20])$. For our purposes, it is enough to consider periodic states only. Let $\Lambda$ stand for a finite subset of the lattice $\mathbf{Z}^{d}$, which we may choose as a cubic box of finite length. For such a box, we define the periodic dynamical matrix

$$
d_{\mathbf{l l}^{\prime}}^{\Lambda}=-\phi\left(\left|\mathbf{l}-\mathbf{l}^{\prime}\right|_{\Lambda}\right),
$$

where the function $\phi$ is the same as in (2) and the periodic distance $\left|\mathbf{l}-\mathbf{l}^{\prime}\right|_{\Lambda}$ is taken on the torus which one obtains identifying the opposite walls of the cube $\Lambda$. The local Hamiltonian $H_{\Lambda}$ corresponding to the periodic boundary conditions is constructed according to (1) and has the following form

$$
H_{\Lambda}=\sum_{\mathbf{l}, \mathbf{1}^{\prime} \in \Lambda} d_{11^{\prime}}^{\Lambda}\left(\mathbf{q}_{\mathbf{l}}, \mathbf{q}_{\mathbf{l}^{\prime}}\right)+\sum_{\mathbf{l} \in \Lambda} H_{\mathbf{l}} .
$$

This Hamiltonian defines a density matrix

$$
\varrho_{\beta, \Lambda}=\exp \left(-\beta H_{\Lambda}\right) / Z_{\beta, \Lambda}, \quad Z_{\beta, \Lambda}=\operatorname{trace} \exp \left(-\beta H_{\Lambda}\right),
$$

where $\beta^{-1}$ is the temperature. For the Hamiltonian (5), a general theory of the Schrödinger operators [12] implies that the latter trace exists. The local periodic Gibbs state $\gamma_{\beta, \Lambda}$ in the box $\Lambda$ is defined as

$$
\gamma_{\beta, \Lambda}(A)=\operatorname{trace}\left\{A \varrho_{\beta, \Lambda}\right\}
$$

where $A$ is an observable, which is a self-adjoint operator representing a certain physical quantity. The state $\gamma_{\beta, \Lambda}$ maps the set of such observables into the real numbers.

The physical properties of the system in $\Lambda$ are described by Matsubara functions, which, for the observables $A_{1}, \ldots, A_{n}$ and $\tau_{1}, \ldots, \tau_{n} \in[0, \beta]$, are defined as follows

$$
\Gamma_{A_{1}, \ldots, A_{n}}^{\beta, \Lambda}\left(\tau_{1}, \ldots, \tau_{n}\right)=\gamma_{\beta, \Lambda}\left\{T_{\tau} h_{\tau_{1}}^{\Lambda}\left(A_{1}\right) \ldots h_{\tau_{n}}^{\Lambda}\left(A_{n}\right)\right\}
$$

where

$$
h_{\tau_{k}}^{\Lambda}\left(A_{k}\right)=\exp \left(-\tau_{k} H_{\Lambda}\right) A_{k} \exp \left(\tau_{k} H_{\Lambda}\right), \quad k=1,2, \ldots, n .
$$

is the Heisenberg representation of the operator $A_{k}$ taken at the imaginary time $t_{k}=\mathrm{i} \tau_{k}$ and $T_{\tau}$ stands for the $\tau$-ordering. By (8), each Matsubara function has the property

$$
\Gamma_{A_{1}, \ldots, A_{n}}^{\beta, \Lambda}\left(\tau_{1}+\theta, \ldots, \tau_{n}+\theta\right)=\Gamma_{A_{1}, \ldots, A_{n}}^{\beta, \Lambda}\left(\tau_{1}, \ldots, \tau_{n}\right), \quad \theta \in[0, \beta]
$$


where addition is understood to be of modulo $\beta$. A significant role in the Euclidean approach is played by multiplication operators. Let $|\Lambda|$ be the number of lattice points in the box $\Lambda$. By $\mathbf{x}_{\Lambda}$ we denote the vector $\mathbf{x}_{\Lambda}=\left(\mathbf{x}_{1}\right)_{\mathbf{l} \in \Lambda}$, which has $|\Lambda|$ components $\mathbf{x}_{\mathbf{l}}$, each of which is a $D$-dimensional vector. For a wave function $\psi=\psi\left(\mathbf{x}_{\Lambda}\right)$ and for a continuous complex valued function $A\left(\mathbf{x}_{\Lambda}\right)$, the corresponding multiplication operator, denoted also as $A$, acts

$$
(A \psi)\left(\mathbf{x}_{\Lambda}\right)=A\left(\mathbf{x}_{\Lambda}\right) \psi\left(\mathbf{x}_{\Lambda}\right) .
$$

Since such multiplication operators commute, the Matsubara functions constructed with them are continuous as functions of $\tau_{1}, \ldots, \tau_{n}$ on the whole cube $[0, \beta]^{n}$.

In the model considered the phase transition is connected with the appearance of the macroscopic displacements of particles breaking $O(D)$-symmetry, thus the operator describing fluctuations of these displacements will play an important role in our consideration. This operator is

$$
Q_{\Lambda}^{(\alpha)}=\frac{1}{\sqrt{|\Lambda|}} \sum_{\mathbf{l} \in \Lambda} q_{\mathbf{l}}^{(\alpha)}, \quad \alpha=1,2, \ldots, D
$$

It is a multiplication operator. The Matsubara function (8) constructed with $n=2$ and $A_{1}=A_{2}=Q_{\Lambda}^{(\alpha)}$ will be in the center of our study. To simplify notations we set

$$
G_{\beta, \Lambda}^{(\alpha)}\left(\tau, \tau^{\prime}\right) \stackrel{\text { def }}{=} \Gamma_{Q_{\Lambda}^{(\alpha)}, Q_{\Lambda}^{(\alpha)}}^{\beta, \Lambda}\left(\tau, \tau^{\prime}\right), \quad \alpha=1,2, \ldots, D .
$$

By (10), this function depends on the distance $\left|\tau-\tau^{\prime}\right|_{\beta} \stackrel{\text { def }}{=} \min \left\{\left|\tau-\tau^{\prime}\right|, \beta-\left|\tau-\tau^{\prime}\right|\right\}$, which makes it possible to write its Fourier transformation in the following form

$$
\begin{aligned}
G_{\beta, \Lambda}^{(\alpha)}\left(\tau, \tau^{\prime}\right) & =\frac{1}{\beta} \sum_{n=-\infty}^{+\infty} \mathrm{e}^{\mathrm{i} \omega_{n}\left(\tau-\tau^{\prime}\right)} \hat{G}_{\beta, \Lambda}^{(\alpha)}\left(\omega_{n}\right) \\
\hat{G}_{\beta, \Lambda}^{(\alpha)}\left(\omega_{n}\right) & =\int_{0}^{\beta} G_{\beta, \Lambda}^{(\alpha)}\left(\tau, \tau^{\prime}\right) \mathrm{e}^{\mathrm{i} \omega_{n}\left(\tau-\tau^{\prime}\right)} \mathrm{d} \tau^{\prime}
\end{aligned}
$$

where $\omega_{n}=(2 \pi / \beta) n, n \in \mathbf{Z}$ are Matsubara's frequencies. The Fourier coefficients $\hat{G}_{\beta, \Lambda}^{(\alpha)}\left(\omega_{n}\right)$ have direct physical meaning - they are susceptibilities of the system in the box $\Lambda$ at the temperature $\beta^{-1}$. For every box $\Lambda$ and every $\beta$, these quantities are well defined. Let $\left\{\Lambda_{l}\right\}_{l \in \mathbf{N}}$ be a sequence of boxes such that, for every $l \in \mathbf{N}$, $\Lambda_{l} \subset \Lambda_{l+1}$ and

$$
\bigcup_{l \in \mathbf{N}} \Lambda_{l}=\mathbf{Z}^{d}
$$

In the sequel we deal with such sequences of boxes only, thus we shall assume that every sequence $\left\{\Lambda_{l}\right\}_{l \in \mathbf{N}}$ has the above properties.

The phase transition in the model considered may be detected as follows. If, for a given $\beta$ and an arbitrary sequence of boxes $\left\{\Lambda_{l}\right\}_{l \in \mathbf{N}}$, all the sequences $\left\{\hat{G}_{\beta, \Lambda_{l}}^{(\alpha)}\left(\omega_{n}\right)\right\}_{l \in \mathbf{N}}$, $n \in \mathbf{Z}$ are bounded, then there is no symmetry breaking at this $\beta$. One can show 
(see below) that all $G_{\Lambda}^{(\alpha)}\left(\tau, \tau^{\prime}\right)$ and all $\hat{G}_{\beta, \Lambda}^{(\alpha)}\left(\omega_{n}\right)$ are nonnegative, thus by (14), one has

$$
\hat{G}_{\beta, \Lambda}^{(\alpha)}\left(\omega_{n}\right) \leqslant \hat{G}_{\beta, \Lambda}^{(\alpha)}(0),
$$

for all $\omega_{n}$. This yields that all the sequences $\left\{\hat{G}_{\beta, \Lambda_{l}}^{(\alpha)}\left(\omega_{n}\right)\right\}_{l \in \mathbf{N}}, n \in \mathbf{Z}$ are bounded if $\left\{\hat{G}_{\beta, \Lambda_{l}}^{(\alpha)}(0)\right\}_{l \in \mathbf{N}}$ is bounded. In this case one says that the fluctuations of displacements of particles are normal. The abnormal fluctuations appear at the critical point of the model or below it (with respect to the temperature, for more details see [39]), they occur if some of the above sequences of susceptibilities become unbounded (divergent). By (15), in all such cases the sequence $\left\{\hat{G}_{\beta, \Lambda_{l}}^{(\alpha)}(0)\right\}_{l \in \mathbf{N}}$ should diverge. Such divergent sequences may be made bounded by multiplying by elements of an infinitely small sequence of numbers, e.g. by $\lambda_{l}=\left|\Lambda_{l}\right|^{-\delta / 2}, \delta>0$. Suppose that, for a divergent sequence $\left\{\hat{G}_{\beta, \Lambda_{l}}^{(\alpha)}\left(\omega_{n}\right)\right\}_{l \in \mathbf{N}}, n \in \mathbf{N}$, one finds $\delta_{n}>0$ and $l_{0} \in \mathbf{N}$ such that

$$
\left\{\left|\Lambda_{l}\right|^{-\delta / 2} \hat{G}_{\beta, \Lambda_{l}}^{(\alpha)}\left(\omega_{n}\right)\right\}_{l \geqslant l_{0}} \subset[a, b]
$$

with certain $0<a<b<\infty$. Then the exponent $\delta_{n}>0$ gives the rate of divergence of the sequence $\left\{\hat{G}_{\beta, \Lambda_{l}}^{(\alpha)}\left(\omega_{n}\right)\right\}_{l \in \mathbf{N}}$, and may be considered as a critical exponent. One can show that the largest value of all such exponents is $\delta=1$. If $\delta_{0}=1$, the spontaneous $O(D)$-symmetry breaking occurs at this $\beta$ and the long range order appears. The smallest limiting point of the sequence (16) with $\omega_{n}=0$ is treated as an order parameter. A detailed discussion of phase transitions, order parameters etc, in quantum spin models may be found in [17]. If, for a given $\beta$, (16) holds with $n=0$ and a certain $\delta_{0} \in(0,1)$, the fluctuations are not strong enough to destroy the symmetry but, being abnormal, they correspond to the critical point of the model and such $\beta$ is the critical inverse temperature for this model. Thus, to prove that the model considered undergoes a phase transition, connected with the appearance of macroscopic displacements of particles, one has to show that (16) holds true (for sufficiently large $\beta$ ) with $n=0$ and $\delta_{0}=1$. This was done, for models of the type of $(1)$, in a number of papers $[10,11,16,21,27]$. Here one has to remark that, for the isotropic ferromagnetic Heisenberg model, the appearance of a long range order has not been proven so far. Another remark is that the existence of the long range order ((16) with $\omega_{n}=0$ and $\left.\delta_{0}=1\right)$ does not mean the existence of the critical point ((16) with a certain $\omega_{n}$ and $\left.\delta_{n} \in(0,1)\right)$.

In this article we are going to show that, for the models considered: (a) (16) never holds for $\omega_{n} \neq 0$ (quantum fluctuations are always normal); (b) (16) does not hold for any $\beta$, if the model is "strongly quantum". To make the latter condition more precise we have to introduce the scalar version of the model (1). Namely, let

$$
\begin{aligned}
\widetilde{H} & =\frac{1}{2} \sum_{\mathbf{1}, \mathbf{I}^{\prime} \in \mathbf{Z}^{d}} d_{\mathbf{l}^{\prime}} q_{\mathbf{1}} q_{\mathbf{1}^{\prime}}+\sum_{\mathbf{l} \in \mathbf{Z}^{d}} \widetilde{H}_{\mathbf{l}}, \\
\widetilde{H}_{\mathbf{l}} & =\frac{1}{2} p_{\mathbf{l}}^{2}+\frac{k}{2} q_{\mathbf{l}}^{2}+V\left(q_{\mathbf{l}}^{2}\right),
\end{aligned}
$$

and the anharmonicity potential $V$ is given by (3). The one-particle Hamiltonian $\widetilde{H}_{\mathbf{l}}$ has a pure point spectrum, each element of which $\epsilon_{s}, s \in \mathbf{N}$ is its non-degenerate 
eigenvalue (see e.g. [12]). We set

$$
\Delta=\min \left\{\epsilon_{s+1}-\epsilon_{s} \mid s \in \mathbf{N}\right\} .
$$

One can expect that, for the double-well potentials, the above $\Delta$ is just the frequency of the tunnelling motion between the wells. Since the model (17) in an evident way is connected with the model (1), the parameter $\Delta$ determines the properties of the latter. In what follows, we will say that the model (1) is strongly quantum if the following condition is satisfied

$$
m \Delta^{2}>-\sum_{\mathbf{l}^{\prime} \in \mathbf{Z}^{d}} d_{\mathbf{l}^{\prime}}=\sum_{\mathbf{l} \in \mathbf{Z}^{d}} \phi(|\mathbf{l}|) \stackrel{\text { def }}{=} J .
$$

Its physical interpretation could be as follows: the total force acting on a given quantum particle from all other particles is less than the energy of its quantum zero oscillations (we use the units with $\hbar=1$ ).

Now we are at a position to formulate our results.

Theorem 1 For the model considered, the sequences $\left\{\hat{G}_{\beta, \Lambda_{l}}^{(\alpha)}\left(\omega_{n}\right)\right\}_{l \in \mathbf{N}}$ with $\omega_{n} \neq 0$ are bounded at all temperatures.

Theorem 2 For the model considered, let the condition (19) be satisfied. Then, for all $d, D \in \mathbf{N}$, for any $\beta>0$, the sequence $\left\{\hat{G}_{\beta, \Lambda_{l}}^{(\alpha)}(0)\right\}_{l \in \mathbf{N}}$ is bounded, which means that the model has no critical points and no symmetry breaking at all temperatures.

The proof of the first theorem is quite simple and we give it right now. The proof of the second one will be performed in the framework of the Euclidean approach based on the representation of the Matsubara functions (8) as functional integrals.

Proof of theorem 1. In fact we are going to prove that, for an arbitrary box $\Lambda$ and for any $\beta$,

$$
0 \leqslant \hat{G}_{\beta, \Lambda}^{(\alpha)}\left(\omega_{n}\right) \leqslant \frac{1}{m \omega_{n}^{2}}, \quad \omega_{n} \neq 0
$$

where $m$ is the particle mass. One can show that also the Hamiltonian (9) has a pure point spectrum, but this time the eigenvalues $\mathcal{E}_{s}, s \in \mathbf{N}$ are degenerate due to the $O(D)$-symmetry (and the corresponding parameter $\Delta$ would be zero in this case). Let $\Psi_{s}, s \in \mathbf{N}$ be the corresponding eigenfunctions of $H_{\Lambda}$. We set

$$
Q_{s s^{\prime}}=\left\langle\Psi_{s}\left|Q_{\Lambda}^{(\alpha)}\right| \Psi_{s^{\prime}}\right\rangle
$$

Then by (8), (12)-(14), one has

$$
\hat{G}_{\beta, \Lambda}^{(\alpha)}\left(\omega_{n}\right)=\frac{1}{Z_{\beta, \Lambda}} \sum_{s, s^{\prime} \in \mathbf{N}} Q_{s s^{\prime}}^{2} \frac{\mathcal{E}_{s}-\mathcal{E}_{s^{\prime}}}{\left(\mathcal{E}_{s}-\mathcal{E}_{s^{\prime}}\right)^{2}+\omega_{n}^{2}}\left[\exp \left(-\beta \mathcal{E}_{s^{\prime}}\right)-\exp \left(-\beta \mathcal{E}_{s}\right)\right]
$$

This immediately yields $\hat{G}_{\beta, \Lambda}^{(\alpha)}\left(\omega_{n}\right) \geqslant 0$. Further, one may estimate the denominator in the above expression

$$
\left(\mathcal{E}_{s}-\mathcal{E}_{s^{\prime}}\right)^{2}+\omega_{n}^{2} \geqslant \omega_{n}^{2}>0
$$


and obtain

$$
\begin{aligned}
\hat{G}_{\beta, \Lambda}^{(\alpha)}\left(\omega_{n}\right) \leqslant & \frac{1}{\omega_{n}^{2} Z_{\beta, \Lambda}} \sum_{s, s^{\prime} \in \mathbf{N}} Q_{s s^{\prime}}^{2}\left(\mathcal{E}_{s}-\mathcal{E}_{s^{\prime}}\right) \\
& \times\left[\exp \left(-\beta \mathcal{E}_{s^{\prime}}\right)-\exp \left(-\beta \mathcal{E}_{s}\right)\right] \\
& =\frac{1}{\omega_{n}^{2}} \gamma_{\beta, \Lambda}\left\{\left[Q_{\Lambda}^{(\alpha)},\left[H_{\Lambda}, Q_{\Lambda}^{(\alpha)}\right]\right]\right\}=\frac{1}{m \omega_{n}^{2}}
\end{aligned}
$$

where $[\ldots, \ldots]$ stands for commutator.

\section{Euclidean approach}

The proof of our second theorem is not so elementary. To prove the boundedness stated we have to have instruments for estimating Matsubara functions (8). These instruments are provided by the Euclidean approach. Its basic element is the representation of the functions (8) constructed with multiplication operators as functional integrals. We begin the description of these integrals with introducing the sets of functions on which we define them. Let $\mathcal{W}_{\beta}$ stand for the set of continuous functions $\mathbf{w}=\mathbf{w}(\tau)=\left(w^{(1)}(\tau), \ldots, w^{(D)}(\tau)\right)$ defined on $[0, \beta]$, taking values in $\mathbf{R}^{D}$ and such that $\mathbf{w}(0)=\mathbf{w}(\beta)$. These functions are called periodic paths. The set of all vectors $\mathbf{w}_{\Lambda}=\left(\mathbf{w}_{\mathbf{l}}\right)_{\mathbf{l} \in \Lambda}$, each component of which is a periodic path at the point $\mathbf{l} \in \Lambda$ is denoted by $\mathcal{W}_{\beta, \Lambda}$. Now we have to construct the measure on $\mathcal{W}_{\beta, \Lambda}$ which will define the functional integral. First we introduce the Gaussian measure $\mathrm{d} \chi_{\beta}$ which we shall use as a reference measure. This measure in determined by the first two terms in $H_{1}$ (1) and describes a vector harmonic oscillator. Since our integration sets $\mathcal{W}_{\beta}, \mathcal{W}_{\beta, \Lambda}$ are infinite dimensional vector spaces, on which Lebesgue's measure $\mathrm{d}$ w does not exist, we cannot write our Gaussian measure in the way density $\times \mathrm{d} \mathbf{w}$, as is usual for the finite dimensional case. Nevertheless, the Gaussian measure is determined by its Fourier transformation, which for our measure $\mathrm{d} \chi_{\beta}$ reads

$$
\begin{aligned}
& \int_{\mathcal{W}_{\beta}} \exp \left(\mathrm{i} \int_{0}^{\beta}(\mathbf{w}(\tau), \mathbf{x}(\tau)) \mathrm{d} \tau\right) \mathrm{d} \chi_{\beta}(\mathbf{w})= \\
& \quad=\exp \left\{-\frac{1}{2} \int_{0}^{\beta} \int_{0}^{\beta}\left(S\left(\tau, \tau^{\prime}\right) \mathbf{x}(\tau), \mathbf{x}\left(\tau^{\prime}\right)\right) \mathrm{d} \tau \mathrm{d} \tau^{\prime}\right\}
\end{aligned}
$$

where

$$
\begin{aligned}
\left(S\left(\tau, \tau^{\prime}\right) \mathbf{x}(\tau), \mathbf{x}\left(\tau^{\prime}\right)\right) & =\sum_{\alpha, \alpha^{\prime}=1}^{D} S^{\alpha \alpha^{\prime}}\left(\tau, \tau^{\prime}\right) x^{(\alpha)}(\tau) x^{\left(\alpha^{\prime}\right)}\left(\tau^{\prime}\right) \\
S^{\alpha \alpha^{\prime}}\left(\tau, \tau^{\prime}\right) & =\delta_{\alpha \alpha^{\prime}} \Omega_{0} \frac{\exp \left[\Omega_{0}\left(\beta-\left|\tau-\tau^{\prime}\right|\right)\right]+\exp \left[\Omega_{0}\left|\tau-\tau^{\prime}\right|\right]}{\exp \left(\Omega_{0} \beta\right)-1}
\end{aligned}
$$

and $\Omega_{0}=\sqrt{k / m}$ is the standard parameter of the harmonic oscillator. All properties of the measure $\mathrm{d} \chi_{\beta}$ are known (see e.g. subsection 2.2 in [6]). By means of it 
we construct the Gaussian measure which describes the system of non-interacting harmonic oscillators located in $\Lambda$ :

$$
\mathrm{d} \chi_{\beta, \Lambda}\left(\mathbf{w}_{\Lambda}\right)=\prod_{\mathbf{l} \in \Lambda} \mathrm{d} \chi_{\beta}\left(\mathbf{w}_{\mathbf{l}}\right) .
$$

Now we take into account the anharmonicity potential and the interaction between the particles. It is performed using the Feynman-Kac formula, the detailed description of which the reader may find in [30] and [32]. In what follows, we introduce the measure

$$
\begin{aligned}
\mathrm{d} \nu_{\beta, \Lambda}\left(\mathbf{w}_{\Lambda}\right)=\frac{1}{Z_{\beta, \Lambda}} \exp \{ & -\frac{1}{2} \sum_{\mathbf{l}, \mathbf{l}^{\prime} \in \Lambda} d_{\mathbf{l}^{\prime}}^{\Lambda} \int_{0}^{\beta}\left(\mathbf{w}_{\mathbf{l}}(\tau), \mathbf{w}_{\mathbf{l}^{\prime}}(\tau)\right) \mathrm{d} \tau \\
& -\sum_{\mathbf{l} \in \Lambda} \int_{0}^{\beta} V\left(\left(\mathbf{w}_{\mathbf{l}}(\tau), \mathbf{w}_{\mathbf{l}}(\tau)\right) \mathrm{d} \tau\right\} \mathrm{d} \chi_{\beta, \Lambda}\left(\mathbf{w}_{\Lambda}\right) .
\end{aligned}
$$

This measure is called a periodic local Euclidean Gibbs measure. As usual, the factor in front of the exp is the normalization constant, which provides that the normalization condition

$$
\int_{\mathcal{W}_{\beta, \Lambda}} \mathrm{d} \nu_{\beta, \Lambda}(\mathbf{w})=1
$$

is satisfied. On the other hand, $Z_{\beta, \Lambda}$ is the partition function of the system in the box $\Lambda$ and has the representation following from (27)

$$
\begin{aligned}
Z_{\beta, \Lambda}=\int_{\mathcal{W}_{\beta, \Lambda}} \exp \{ & -\frac{1}{2} \sum_{\mathbf{l}, \mathbf{l}^{\prime} \in \Lambda} d_{\mathbf{l}^{\prime}}^{\Lambda} \int_{0}^{\beta}\left(\mathbf{w}_{\mathbf{l}}(\tau), \mathbf{w}_{\mathbf{l}^{\prime}}(\tau)\right) \mathrm{d} \tau \\
& -\sum_{\mathbf{l} \in \Lambda} \int_{0}^{\beta} V\left(\left(\mathbf{w}_{\mathbf{l}}(\tau), \mathbf{w}_{\mathbf{l}}(\tau)\right) \mathrm{d} \tau\right\} \mathrm{d} \chi_{\beta, \Lambda}\left(\mathbf{w}_{\Lambda}\right)
\end{aligned}
$$

Now we are at a position to write the main formula derived in [2]. It is

$$
\Gamma_{A_{1}, \ldots, A_{n}}^{\beta, \Lambda}\left(\tau_{1}, \ldots, \tau_{n}\right)=\int_{\mathcal{W}_{\beta, \Lambda}} A_{1}\left(\mathbf{w}_{\Lambda}\left(\tau_{1}\right)\right) \cdots A_{1}\left(\mathbf{w}_{\Lambda}\left(\tau_{n}\right)\right) \mathrm{d} \nu_{\beta, \Lambda}\left(\mathbf{w}_{\Lambda}\right),
$$

where $A_{1}, \ldots, A_{n}$ are the multiplication operators by the functions $A_{1}\left(\mathbf{x}_{\Lambda}\right), \ldots$, $A_{n}\left(\mathbf{x}_{\Lambda}\right)$ respectively. This representation is employed to obtain information regarding mathematical properties of the Matsubara functions and their sequences. It is worth noting that representations of this kind may be used (and are used) as a base in the study of such models by means of computer simulations (see e.g. [33] and the references therein).

Obviously, the same construction may be also performed for the scalar Hamiltonian (17). This will yield the local Euclidean Gibbs measure $\mathrm{d} \tilde{\nu}_{\beta, \Lambda}\left(w_{\Lambda}\right)$

$$
\mathrm{d} \tilde{\nu}_{\beta, \Lambda}\left(w_{\Lambda}\right)=\frac{1}{\widetilde{Z}_{\beta, \Lambda}} \exp \left\{-\frac{1}{2} \sum_{\mathbf{l}, \mathbf{I}^{\prime} \in \Lambda} d_{\mathbf{l l}^{\prime}}^{\Lambda} \int_{0}^{\beta} w_{\mathbf{l}}(\tau) w_{\mathbf{l}^{\prime}}(\tau) \mathrm{d} \tau\right.
$$




$$
\left.-\sum_{\mathbf{l} \in \Lambda} \int_{0}^{\beta} V\left(w_{\mathbf{l}}^{2}(\tau)\right) \mathrm{d} \tau\right\} \mathrm{d} \tilde{\chi}_{\beta, \Lambda}\left(w_{\Lambda}\right)
$$

where the Gaussian measure $\mathrm{d} \tilde{\chi}_{\beta, \Lambda}\left(w_{\Lambda}\right)$ is defined by $(25)$ with $\mathrm{d} \chi_{\beta}\left(\mathbf{w}_{\mathbf{l}}\right)$ replaced by $\mathrm{d} \tilde{\chi}_{\beta}\left(w_{1}\right)$. The latter measure is defined by (22)-(24) with $\alpha=\alpha^{\prime}=1$. The corresponding space of scalar paths will be denoted $\widetilde{\mathcal{W}}_{\beta, \Lambda}$. Accordingly, the Matsubara functions given by the integrals (29) with $\nu$ replaced by $\tilde{\nu}$ will be written $\widetilde{\Gamma}$.

Let us consider the set of continuous real valued functions $\widetilde{\mathcal{F}}_{\Lambda}$ defined on the set $\mathbf{R}^{|\Lambda|}=\left\{x_{\Lambda}=\left(x_{1}\right)_{\mathbf{l} \in \Lambda} \mid x_{\mathbf{l}} \in \mathbf{R}, \quad \mathbf{l} \in \Lambda\right\}$ which have the following properties. A function $A: \mathbf{R}^{|\Lambda|} \rightarrow \mathbf{R}$ belongs to $\widetilde{\mathcal{F}}_{\Lambda}$ if: (a) there exist $\mathbf{l} \in \Lambda$ such that $A$ depends on $x_{1}$ only; (b) this dependence is either odd monotone or even positive and monotone on $\mathbf{R}_{+}$. The basic element of the proof of theorem 2 is the following assertion, proved in [23], see also [6], section 7 .

Proposition 3 [Scalar Domination] Let the continuous functions $A_{1}, \ldots, A_{n}$ : $\mathbf{R}^{D|\Lambda|} \rightarrow \mathbf{R}$ have the following property: there exist $\alpha \in\{1,2, \ldots, D\}$ and the functions $\widetilde{A}_{1}, \ldots, \widetilde{A}_{n} \in \widetilde{\mathcal{F}}_{\Lambda}$ such that $A_{j}\left(\mathbf{x}_{\Lambda}\right)=\widetilde{A}_{j}\left(x_{\Lambda}^{(\alpha)}\right), j=1,2, \ldots, n$. Then for arbitrary $D \in \mathbf{N}$ and for all $\tau_{1}, \ldots, \tau_{n} \in[0, \beta]$,

$$
0 \leqslant \Gamma_{A_{1}, \ldots, A_{n}}^{\beta, \Lambda}\left(\tau_{1}, \ldots, \tau_{n}\right) \leqslant \widetilde{\Gamma}_{\widetilde{A}_{1}, \ldots, \widetilde{A}_{n}}^{\beta, \Lambda}\left(\tau_{1}, \ldots, \tau_{n}\right) .
$$

Let us stress that all $A_{j}, j=1, \ldots, n$ above are supposed to depend on their $x_{\Lambda}^{(\alpha)}$ with one and the same $\alpha$. As a corollary of this statement we have

$$
0 \leqslant G_{\beta, \Lambda}^{(\alpha)}\left(\tau, \tau^{\prime}\right) \leqslant \widetilde{G}_{\beta, \Lambda}\left(\tau, \tau^{\prime}\right)
$$

where the function $\widetilde{G}_{\beta, \Lambda}\left(\tau, \tau^{\prime}\right)$ is defined by (13) but with $Q_{\Lambda}^{(\alpha)}(12)$ replaced by

$$
\widetilde{Q}_{\Lambda}=\frac{1}{\sqrt{|\Lambda|}} \sum_{\mathbf{l} \in \Lambda} q_{\mathbf{l}}
$$

and with the Matsubara function $\Gamma$ replaced by $\widetilde{\Gamma}$. Thus, in order to prove the boundedness of $\left\{G_{\beta, \Lambda_{l}}^{(\alpha)}\right\}_{l \in \mathbf{N}}$ it is enough to prove it for $\left\{\widetilde{G}_{\beta, \Lambda_{l}}\right\}_{l \in \mathbf{N}}$. To this end we introduce the following local Gibbs measure. For $t \in[0,1]$, we set

$$
\begin{aligned}
\mathrm{d} \tilde{\nu}_{\beta, \Lambda}^{(t)}\left(w_{\Lambda}\right)=\frac{1}{\widetilde{Z}_{\beta, \Lambda}(t)} \exp \{ & -\frac{t}{2} \sum_{\mathbf{l}, \mathbf{I}^{\prime} \in \Lambda} d_{\mathbf{l l}^{\prime}}^{\Lambda} \int_{0}^{\beta} w_{\mathbf{l}}(\tau) w_{\mathbf{l}^{\prime}}(\tau) \mathrm{d} \tau \\
& \left.-\sum_{\mathbf{l} \in \Lambda} \int_{0}^{\beta} V\left(w_{\mathbf{l}}^{2}(\tau)\right) \mathrm{d} \tau\right\} \mathrm{d} \tilde{\chi}_{\beta, \Lambda}\left(w_{\Lambda}\right),
\end{aligned}
$$

where $\widetilde{Z}_{\beta, \Lambda}(t)$ is the corresponding normalization constant defined by $(27)$, which may be written similarly to (28). By means of this measure we define (see (29), (13), $(12),(9))$

$$
\widetilde{G}_{\beta, \Lambda}\left(\tau, \tau^{\prime} \mid t\right)=\int_{\widetilde{\mathcal{W}}_{\beta, \Lambda}}\left(\frac{1}{\sqrt{|\Lambda|}} \sum_{\mathbf{l} \in \Lambda} w_{\mathbf{l}}(\tau)\right)\left(\frac{1}{\sqrt{|\Lambda|}} \sum_{\mathbf{l} \in \Lambda} w_{\mathbf{l}}\left(\tau^{\prime}\right)\right) \mathrm{d} \tilde{\nu}_{\beta, \Lambda}^{(t)}\left(w_{\Lambda}\right) .
$$


Clearly

$$
\widetilde{G}_{\beta, \Lambda}\left(\tau, \tau^{\prime} \mid 1\right)=\widetilde{G}_{\beta, \Lambda}\left(\tau, \tau^{\prime}\right)
$$

and (see (8) and (7))

$$
\begin{aligned}
\widetilde{G}_{\beta, \Lambda}\left(\tau, \tau^{\prime} \mid 0\right)= & \frac{1}{|\Lambda|} \sum_{\mathbf{l} \in \Lambda} \frac{1}{\widetilde{Z}_{\beta, 1}} \operatorname{trace}\left\{q_{\mathbf{l}} \exp \left(-\left(\tau-\tau^{\prime}\right) \widetilde{H}_{1}\right)\right. \\
& \left.\times q_{1} \exp \left(-\left(\beta-\tau+\tau^{\prime}\right) \widetilde{H}_{1}\right)\right\} \stackrel{\text { def }}{=} U\left(\tau, \tau^{\prime}\right) .
\end{aligned}
$$

Here we have taken into account that the traces in the latter formula do not depend on $\mathbf{l}$ due to the translation invariance of the one-particle Hamiltonian (17). We also set

$$
\begin{aligned}
& R_{\mathbf{l}_{1} \mathbf{l}_{\mathbf{2}} \mathbf{l}_{\mathbf{l}_{4}} \mathbf{l}_{4}}\left(\tau_{1}, \tau_{2}, \tau_{3}, \tau_{4} \mid t\right)= \\
& =\int_{\widetilde{\mathcal{W}}_{\beta, \Lambda}} w_{\mathbf{l}_{1}}\left(\tau_{1}\right) w_{\mathbf{l}_{2}}\left(\tau_{2}\right) w_{\mathbf{l}_{3}}\left(\tau_{3}\right) w_{\mathbf{l}_{4}}\left(\tau_{4}\right) \mathrm{d} \tilde{\nu}_{\beta, \Lambda}^{(t)}\left(w_{\Lambda}\right) \\
& \quad-\int_{\widetilde{\mathcal{W}}_{\beta, \Lambda}} w_{\mathbf{l}_{1}}\left(\tau_{1}\right) w_{\mathbf{l}_{2}}\left(\tau_{2}\right) \mathrm{d} \tilde{\nu}_{\beta, \Lambda}^{(t)}\left(w_{\Lambda}\right) \times \int_{\widetilde{\mathcal{W}}_{\beta, \Lambda}} w_{\mathbf{l}_{3}}\left(\tau_{3}\right) w_{\mathbf{l}_{4}}\left(\tau_{4}\right) \mathrm{d} \tilde{\nu}_{\beta, \Lambda}^{(t)}\left(w_{\Lambda}\right) \\
& \quad-\int_{\widetilde{\mathcal{W}}_{\beta, \Lambda}} w_{\mathbf{l}_{1}}\left(\tau_{1}\right) w_{\mathbf{l}_{3}}\left(\tau_{3}\right) \mathrm{d} \tilde{\nu}_{\beta, \Lambda}^{(t)}\left(w_{\Lambda}\right) \times \int_{\widetilde{\mathcal{W}}_{\beta, \Lambda}} w_{\mathbf{l}_{2}}(\tau) w_{\mathbf{l}_{4}}\left(\tau_{4}\right) \mathrm{d} \tilde{\nu}_{\beta, \Lambda}^{(t)}\left(w_{\Lambda}\right) \\
& \quad-\int_{\widetilde{\mathcal{W}}_{\beta, \Lambda}} w_{\mathbf{l}_{1}}\left(\tau_{1}\right) w_{\mathbf{l}_{4}}\left(\tau_{4}\right) \mathrm{d} \tilde{\nu}_{\beta, \Lambda}^{(t)}\left(w_{\Lambda}\right) \times \int_{\widetilde{\mathcal{W}}_{\beta, \Lambda}} w_{\mathbf{l}_{2}}\left(\tau_{2}\right) w_{\mathbf{l}_{3}}\left(\tau_{3}\right) \mathrm{d} \tilde{\nu}_{\beta, \Lambda}^{(t)}\left(w_{\Lambda}\right)
\end{aligned}
$$

One can show that all $\widetilde{G}_{\beta, \Lambda}$ and $R_{\beta, \Lambda}$, as functions of $t \in[0,1]$, are differentiable on $t \in(0,1)$ and continuous on $t \in[0,1]$. Furthermore,

$$
R_{\mathbf{l}_{1} \mathbf{l}_{2} \mathbf{l}_{3} \mathbf{l}_{4}}^{\beta, \Lambda}\left(\tau_{1}, \tau_{2}, \tau_{3}, \tau_{4} \mid t\right) \leqslant 0,
$$

for all values of its arguments. This estimate is known as Lebowitz' inequality, its validity for the measure (30) was proved in section 6 of [6]. Differentiating both sides of (34) with respect to $t$ after some calculations we obtain

$$
\begin{aligned}
& \frac{\partial}{\partial t} \widetilde{G}_{\beta, \Lambda}\left(\tau_{1}, \tau_{2} \mid t\right)=-\frac{1}{2|\Lambda|} \sum_{\mathbf{l}_{1}, \ldots, \mathbf{l}_{4} \in \Lambda} d_{\mathbf{l}_{1} \mathbf{l}_{2}}^{\Lambda} \int_{0}^{\beta} R_{\mathbf{l}_{1} \mathbf{l}_{2} \mathbf{l}_{3} \mathbf{l}_{4}}^{\beta, \Lambda}\left(\tau_{1}, \tau_{2}, \tau, \tau \mid t\right) \mathrm{d} \tau \\
& +\left(-\sum_{\mathbf{l} \in \Lambda} d_{\mathbf{l l}^{\prime}}^{\Lambda}\right) \int_{0}^{\beta} \widetilde{G}_{\beta, \Lambda}\left(\tau_{1}, \tau \mid t\right) \widetilde{G}_{\beta, \Lambda}\left(\tau, \tau_{2} \mid t\right) \mathrm{d} \tau
\end{aligned}
$$

Here we have taken into account the translation invariance of the moments of the measure (33), which is connected with the invariance of the dynamic matrix $d_{11^{\prime}}^{\Lambda}$. The above formula may be considered as an integro-differential equation with respect to $\widetilde{G}_{\beta, \Lambda}$ subject to the initial condition (36). By (15), which holds also for the scalar model, to prove the boundedness we need it to be sufficient to control the sequences of the following functions

$$
g_{\beta, \Lambda}(t) \stackrel{\text { def }}{=} \int_{0}^{\beta} \widetilde{G}_{\beta, \Lambda}\left(\tau_{1}, \tau_{2} \mid t\right) \mathrm{d} \tau_{2}=\int_{0}^{\beta} \widetilde{G}_{\beta, \Lambda}(0, \tau \mid t) \mathrm{d} \tau .
$$


For this function, we obtain from (39) the following equation

$$
\begin{aligned}
\frac{\partial}{\partial t} g_{\beta, \Lambda}(t)= & -\frac{1}{2|\Lambda|} \sum_{\mathbf{l}_{1}, \ldots, \mathbf{l}_{4} \in \Lambda} d_{\mathbf{l}_{1} \mathbf{l}_{2}}^{\Lambda} \int_{0}^{\beta} \int_{0}^{\beta} R_{\mathbf{l}_{1}, \mathbf{l}_{2} \mathbf{l}_{3}, \mathbf{l}_{4}}^{\beta,}\left(\tau_{1}, \tau_{2}, \tau, \tau \mid t\right) \mathrm{d} \tau \mathrm{d} \tau_{1} \\
& +\left(-\sum_{\mathbf{l} \in \Lambda} d_{\mathbf{l l}^{\prime}}^{\Lambda}\right)\left[g_{\beta, \Lambda}(t)\right]^{2}
\end{aligned}
$$

subject to the initial condition (see (36))

$$
g_{\beta, \Lambda}(0)=u \stackrel{\text { def }}{=} \int_{0}^{\beta} U(0, \tau) \mathrm{d} \tau
$$

Taking into account the assumed properties of the dynamical matrix $d_{\mathbf{l l}^{\prime}}$ one concludes that, for every box $\Lambda$,

$$
\left(-\sum_{\mathbf{l} \in \Lambda} d_{\mathbf{l 1}^{\prime}}^{\Lambda}\right) \leqslant\left(-\sum_{\mathbf{l} \in \mathbf{Z}^{d}} d_{\mathbf{l}^{\prime}}\right)=J>0 .
$$

Let us consider the following differential equation

$$
\frac{\mathrm{d} h(t)}{\mathrm{dt}}=J[h(t)]^{2}, \quad t \in[0,1], \quad h(0)=u .
$$

If the condition

$$
u J<1 \text {, }
$$

is satisfied, it has a unique solution on $t \in[0,1]$, which has the form

$$
h(t)=\frac{u}{1-t u J}
$$

Now let us compare the equations (44) and (41). By (38), the first term in (41) is nonpositive since all $d_{\mathbf{l l}^{\prime}}^{\Lambda}$ are nonpositive. Both our equations are subject to the same initial condition. Applying standard comparison methods of the theory of differential equations (see e.g. [41]) one obtains

$$
\frac{\partial}{\partial t} g_{\beta, \Lambda}(t) \leqslant \frac{\mathrm{d} h(t)}{\mathrm{dt}}
$$

for all $t \in(0,1)$. This immediately yields $g_{\beta, \Lambda}(t) \leqslant h(t)$, for $t \in[0,1]$, in particular

$$
\int_{0}^{\beta} \widetilde{G}_{\beta, \Lambda}(0, \tau) \mathrm{d} \tau=g_{\beta, \Lambda}(1) \leqslant h(1)=\frac{u}{1-u J}
$$

which holds for all boxes $\Lambda$. Here we have also taken into account (35).

Proof of theorem 2. By (14), (32) and (47), the sequence $\left\{\hat{G}_{\beta, \Lambda_{l}}^{(\alpha)}(0)\right\}_{l \in \mathbf{N}}$ is bounded provided (45) holds. Thus, to complete the proof one has to show that the 
condition (19) does imply (45). To this end we employ the method already used in the proof of our first theorem. By (42), (36) and (18), one has

$$
\begin{aligned}
u= & \frac{1}{\widetilde{Z}_{\beta}} \int_{0}^{\beta} \operatorname{trace}\left\{q_{1} \mathrm{e}^{-\tau \widetilde{H}_{1}} q_{1} \mathrm{e}^{-(\beta-\tau) \widetilde{H}_{1}}\right\} \mathrm{d} \tau \\
& =\frac{1}{\widetilde{Z}_{\beta}} \sum_{s, s^{\prime} \in \mathbf{N}}\left[\left\langle\psi_{s}\left|q_{1}\right| \psi_{s^{\prime}}\right\rangle\right]^{2} \frac{\mathrm{e}^{-\beta \epsilon_{s}}-\mathrm{e}^{-\beta \epsilon_{s^{\prime}}}}{\epsilon_{s}-\epsilon_{s^{\prime}}} \\
\leqslant & \frac{1}{\Delta^{2}} \frac{1}{\widetilde{Z}_{\beta}} \sum_{s, s^{\prime} \in \mathbf{N}}\left[\left\langle\psi_{s}\left|q_{1}\right| \psi_{s^{\prime}}\right\rangle\right]^{2}\left[\mathrm{e}^{-\beta \epsilon_{s}}-\mathrm{e}^{-\beta \epsilon_{s^{\prime}}}\right]\left[\epsilon_{s}-\epsilon_{s^{\prime}}\right] \\
= & \frac{1}{\Delta^{2}} \cdot \frac{\operatorname{trace}\left(\left[q_{1},\left[\widetilde{H}_{1}, q_{1}\right]\right] \mathrm{e}^{-\beta \widetilde{H}_{1}}\right)}{\operatorname{trace} \mathrm{e}^{-\beta \widetilde{H}_{1}}}=\frac{1}{m \Delta^{2}} .
\end{aligned}
$$

Here $\psi_{s}, s \in \mathbf{N}$ are the eigenfunctions of $\widetilde{H}_{1}$ corresponding to the eigenvalues $\epsilon_{s}$. Above, in the sums $\sum_{s, s^{\prime}}$ the terms with coinciding $s, s^{\prime}$ give zero contribution because of the corresponding matrix elements of $q_{1}$ vanishing. In deriving the above estimate we have taken into account that the eigenvalues $\epsilon_{s}$ are simple, which is not the case for $D>1$. The latter fact gives the reason why we use our scalar domination arguments.

Since the right-hand side in (47) is a monotone function of $u$, we apply there the latter estimate and obtain

$$
\hat{G}_{\beta, \Lambda}^{(\alpha)}(0) \leqslant \int_{0}^{\beta} \widetilde{G}_{\beta, \Lambda}(0, \tau) \mathrm{d} \tau \leqslant \frac{1}{m \Delta^{2}-J}
$$

which completes the proof.

\section{Discussion}

First let us turn to the estimate (20). Since $\omega_{n}=(2 \pi / \beta) n, n \in \mathbf{Z}$, the series

$$
\sum_{n \in \mathbf{Z} \backslash\{0\}} \hat{G}_{\beta, \Lambda}^{(\alpha)}\left(\omega_{n}\right)
$$

converges for any box $\Lambda$ and for every $\beta>0$. This fact has a deep mathematical consequence - by Ascoli's theorem (see e.g. [28], p. 72), the sequences of Matsubara functions $\left\{\Gamma_{A_{1}, \ldots, A_{n}}^{\beta, \Lambda_{l}}\right\}_{l \in \mathbf{N}}$ (for all multiplication operators $A_{1}, \ldots, A_{n}$, for which they make sense) converge uniformly on $[0, \beta]^{n}$ provided the sequence $\left\{\hat{G}_{\beta, \Lambda_{l}}^{(\alpha)}(0)\right\}_{l \in \mathbf{N}}$ is bounded. This convergence of the Matsubara functions imply in turn that the limiting Gibbs measure, which describes the equilibrium state of the whole anharmonic crystal, exists in this case. It is called periodic Euclidean Gibbs measure (see $[9])$. The boundedness of the sequence $\left\{\hat{G}_{\beta, \Lambda_{l}}^{(\alpha)}(0)\right\}_{l \in \mathbf{N}}$ means the absence of abnormal fluctuations, it may be obtained by satisfying the condition (19), as follows from theorem 2 . The above fact, however, does not imply that there exists the only 
one limiting Gibbs measure (the latter would mean that there exists just one phase and no phase transitions may occur). In fact, the uniqueness of the limiting Gibbs measures may be proved based on the more sophisticated methods. Here one has to mention that such a uniqueness was proved in [5] to hold for $D=1$ and for the values of the particle mass from the interval $\left(0, m_{*}(\beta)\right)$, where the bound $m_{*}(\beta)$ tended to zero as $\beta \rightarrow+\infty$. In a very recent paper [7] it is stated that the uniqueness of the limiting Gibbs measures (again for $D=1$ only) may be guaranteed by a condition similar to (19), which holds for $m \in\left(0, m_{*}\right)$, where $m_{*}$ is independent of $\beta$. In the vector case $D>1$, no uniqueness results have been obtained so far.

Now, let us say some words about the condition (19). In the harmonic case $V=0$, $\Delta=\Omega_{0}$, thus $m \Delta^{2}=k$ and this condition means $k>J$. For the potential $V$ given by (3), one can show that there exists $C>0$ such that $m \Delta^{2} \sim C m^{-(p-1) /(p+1)}$ as $m \rightarrow 0$, which means that the condition (19) is satisfied for $m \in\left(0, m_{*}\right)$, where the bound $m_{*}$ depends on the parameters $J$ and $a, b_{2}, \ldots, b_{p}$ only.

\section{Acknowledgement}

This work was financially supported by the Deutsche Forschungsgemeinschaft through the German-Polish project 436 POL 113/98/0-1 "Probability Measures" which is gratefully acknowledged. The author is also grateful for kind hospitality extended to him at the Forschungszentrum BiBoS, Universität Bielefeld in JuneJuly 2002.

\section{References}

1. Aksenov V.L., Plakida N.M., Stamenković S. Neutron Scattering by Ferroelectrics. Singapure, World Scientific, 1990.

2. Albeverio S., Høegh-Krohn R. Homogeneous random fields and quantum statistical mechanics. // J. Funct. Anal., 1975, vol. 19, p. 242-279.

3. Albeverio S., Høegh-Krohn R. Mathematical Theory of Feynman Path Integrals. Lecture Notes in Math. 523, Berlin New York, Springer, 1976.

4. Albeverio S., Kondratiev Yu., Kozitsky Yu. Suppression of critical fluctuations by strong quantum effects in quantum lattice systems. // Comm. Math. Phys., 1998, vol. 194, p. 493-512.

5. Albeverio S., Kondratiev Yu., Kozitsky Yu., Röckner M. Uniqueness for Gibbs measures of quantum lattices in small mass regime. // Ann. Inst. H. Poincaré, Probab. Statist., 2001, vol. 37, p. 43-69.

6. Albeverio S., Kondratiev Yu., Kozitsky Yu., Röckner M. Euclidean Gibbs states of quantum lattice systems. Preprint BiBoS Nr. 01-03-034, Bielefeld, 2001 (to appear in Rev. Math. Phys.).

7. Albeverio S., Kondratiev Yu., Kozitsky Yu., Röckner M. Gibbs states of a quantum crystal: uniqueness by small particle mass. // C. R. Acad. Sci. Paris, Ser. I, 2002, vol. 335, p. 693-698.

8. Barbulyak V.S., Kondratiev Yu.G. Functional integrals and quantum lattice systems: I. existence of Gibbs states. // Rep. Nat. Acad. Sci of Ukraine, 1991, No. 9, p. 38-40. 
9. Barbulyak V.S., Kondratiev Yu.G. Functional integrals and quantum lattice systems: II. periodic Gibbs states. // Rep. Nat. Acad. Sci of Ukraine, 1991, No. 8, p. 31-34.

10. Barbulyak V.S., Kondratiev Yu.G. Functional integrals and quantum lattice systems: III. phase transitions // Rep. Nat. Acad. Sci of Ukraine, 1991, No. 10, p. 19-21.

11. Barbulyak V.S., Kondratiev Yu.G. The quasiclassical limit for the Schrödinger operator and phase transitions in quantum statistical physics. // Func. Anal. Appl. 1992, vol. 26(2), p. 61-64.

12. Berezin F.A., Shubin M.A. The Schrödinger Equation. Dordrecht Boston London, Kluwer Academic Publishers, 1991.

13. Blinc R., Žekš B. Soft Modes in Ferroelectrics and Antiferroelectrics. Amsterdam, Noth-Holland Publishers, 1974.

14. Bruce A.D., Cowley R.A. Structural Phase Transitions. Taylor and Francis Ltd., 1981.

15. Choquard Ph. The Anharmonic Crystal. New York, W.A. Benjamin, 1967.

16. Driessler W., Landau L., Perez J.F. Estimates of critical lengths and critical temperatures for classical and quantum lattice systems. // J. Stat. Phys. 1979, vol. 20, p. $123-162$.

17. Dyson F.J., Lieb E.H., Simon B. Phase transitions in quantum spin systems with isotropic and nonisotropic interactions. // J. Stat. Phys. 1978, vol. 18, p. 335-383.

18. Freericks J.K., Jarrell M., Mahan G.D. The anharmonic electron-phonon problem. // Phys. Rev. Lett., 1996, vol. 77, p. 4588-4591.

19. Georgii H.-O. Gibbs Measures and Phase Transitions. Vol. 9. New York Berlin, Walter de Gruyter, Springer, 1988.

20. Globa S.A., Kondratiev Yu.G. The construction of Gibbs states of quantum lattice systems. // Selecta Math. Sov., 1990, vol. 9, p. 297-307.

21. Kondratiev Ju.G. Phase transitions in quantum models of ferroelectrics. - In: Stochastic Processes, Physics, and Geometry, vol. 2. Singapore New Jersey, World Scientific, 1994, p. 465-475.

22. Kozitsky Yu. Quantum effects in a lattice model of anharmonic vector oscillators. // Lett. Math. Phys. 2000, vol. 51, p. 71-81.

23. Kozitsky Yu. Scalar domination and normal fluctuation in $N$-vector quantum anharmonic crystals. // Lett. Math. Phys. 2000, vol. 53, p. 289-303.

24. Kozitsky Yu. Quantum effects in lattice models of vector anharmonic oscillators. In: Stochastic Processes, Physics and Geometry: New Interplays, vol. 2, Leipzig, 1999, p. 403-411, CMS Conf. Proc., 29, Amer. Math. Soc., Providence, RI, 2000.

25. Kozitsky Yu. Gibbs states of a lattice systems of quantum anharmonic oscillators. In: Noncommutative Structures in Mathematics and Physics, Kiev, 2000, p. 415-425, (S. Duplij and J. Wess eds) NATO Sci Ser. II Math. Phys. Chem. 22, Kluwer Acad. Publ. Dordrecht, 2001.

26. Kozitsky Yu.V., Levitskii R.R., Stasyuk, I.V. Polariton states in the order-disorder type ferroelectrics. // Theor. Math. Phys. 1979, vol. 39, p. 106-117.

27. Pastur L.A., Khoruzhenko, B.A. Phase transitions in quantum models of rotators and ferroelectrics. // Theor. Math.Phys. 1987, vol. 73, p. 111-124.

28. Mujica J. Complex Analysis in Banach Spaces. Amsterdam New York Oxford, North Holland, 1986.

29. Plakida N.M., Tonchev M.S. Quantum effects in a $d$-dimensional exactly solvable model for a structural phase transition. // Phys. A, 1986, vol. 136, p. 176-188.

30. Roepstorff G. Path Integral Approach to Quantum Physics. Berlin Heidelberg, 
Springer-Verlag, 1993.

31. Schneider T., Beck H., Stoll E. Quantum effects in an $n$-component vector model for structural phase transitions. // Phys. Rev. 1976, vol. B13, p. 1123-1130.

32. Simon B. Functional Integration and Quantum Physics. New York San Francisco London, Academic Press, 1979.

33. Sokal A. Monte Carlo methods in statistical mechanics: foundations and new algorythms. - In: Functional Integration, Cargèse, 1996, p. 131-192, NATO Adv. Sci. Inst. Ser. B Phys., 361, New York, Plenum, 1997.

34. Stamenković S. Unified model description of order-disorder and displascive structural phase transitions. // Condens. Matter Phys., 1998, vol. 1, No. 2(14), p. 257-309.

35. Stasyuk I.V. Local anharmonic effects in high- $T_{\mathrm{c}}$ superconductors. Pseudospinelectron model. // Condens. Matter Phys., 1999, vol. 2(19), p. 435-446.

36. Stasyuk I.V. Approximate analitical dynamical mean-field approach to strongly correlated electron systems. // Condens. Matter Phys., 2000, vol. 3(22), p. 437-456.

37. Tibballs J.E., Nelmes R.J., McIntyre G.J. The crystal structure of tetragonal $\mathrm{KH}_{2} \mathrm{PO}_{4}$ and $\mathrm{KD}_{2} \mathrm{PO}_{4}$ as a function of temperature and pressure. // J. Phys. C: Solid State Phys. 1982, vol. 15 , p. $37-58$.

38. Vaks V.G. Introduction to the Macroscopic Theory of Ferroelectrics. Moscow, Nauka, 1973 (in Russian).

39. Verbeure A., Zagrebnov V.A. Phase transitions and algebra of fluctuation operators in exactly soluble model of a quantum anharmonic crystal. // J. Stat. Phys. 1992, vol. 69 , p. 37-55.

40. Verbeure A., Zagrebnov V.A. No-go theorem for quantum structural phase transition. // J. Phys. A: Math. Gen. 1995, vol. 28, p. 5415-5421.

41. Walter W. Differential and Integral Inequalities. Berlin Heidelberg New York, Springer-Verlag, 1970. 


\section{Квантові ефекти в ангармонічному кристалі}

\section{Ю.Козицький}

Інститут математики, університет ім. Марії Кюрі-Склодовської Польща, 20-031 Люблін

Отримано 30 серпня 2002 р.

Розглядається модель квантових частинок, які виконують $D$-вимірні коливання довкола їх положень рівноваги, що утворюють $d$-вимірну просту кубічну ґратку $\mathbf{Z}^{d}$. Ця модель зазнає фазового переходу, коли флуктуації зміщень частинок стають макроскопічними. Таке явище описується сприйнятливостями, залежними від мацубарівських частот $\omega_{n}, n \in \mathbf{Z}$. Ми доводимо дві теореми, що описують термодинамічні властивості цих сприйнятливостей. Перша теорема стверджує, що сприйнятливості з ненульовими $\omega_{n}$ залишаються обмеженими при всіх температурах, а це означає, що макроскопічні флуктуації в даній моделі є завжди неквантові. Друга теорема дає достатню умову на те, щоб і статична сприйнятливість (яка відповідає $\omega_{n}=0$ ) теж була обмеженою при всіх температурах. Ця умова включає в себе масу частинки, параметри ангармонізму та інтенсивність взаємодії. Фізичний сенс цього результату полягає в тому, що для всіх $D$ і для всіх значень температури сильні квантові ефекти унеможливлюють виникнення критичних точок і далекого порядку. Доведення проводиться в рамках підходу, у якому сприйнятливості представляються за допомогою функціональних інтегралів. Дається короткий опис головних аспектів цього підходу.

Ключові слова: зміщення, структурний фазовий перехід, критична точка

PACS: 05.50.- $-d, 64.60 .-i$ 